\title{
Transmission amplitudes from Bethe ansatz equations
}

\author{
Anastasia Doikou $^{a}$ and Nikos Karaiskos ${ }^{b}$ \\ ${ }^{a}$ Department of Engineering Sciences, University of Patras, \\ GR-26500 Patras, Greece \\ ${ }^{b}$ Institut für Theoretische Physik, Leibniz Universität Hannover, \\ Appelstraße 2, 30167 Hannover, Germany \\ E-mail: adoikou@upatras.gr, nikolaos.karaiskos@itp.uni-hannover.de
}

ABSTRACT: We consider the Heisenberg spin chain in the presence of integrable spin defects. Using the Bethe ansatz methodology, we extract the associated transmission amplitudes, that describe the interaction between the particle-like excitations displayed by the models and the spin impurity. In the attractive regime of the XXZ model, we also derive the breather's transmission amplitude. We compare our findings with earlier relevant results in the context of the sine-Gordon model.

KEYwords: Lattice Integrable Models, Integrable Hierarchies

ArXiv EPRINT: 1212.0195 


\section{Contents}

1 Introduction 1

2 The isotropic case: XXX model 2

2.1 The transmission matrix 4

3 The anisotropic case: XXZ model $\quad 8$

3.1 The repulsive regime; the transmission matrix 9

$\begin{array}{ll}3.2 \text { The attractive regime } & 11\end{array}$

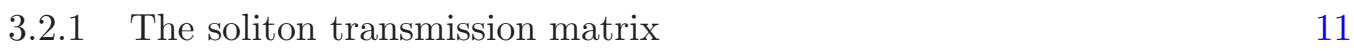

$\begin{array}{ll}\text { 3.2.2 The breather transmission amplitude } & 14\end{array}$

$\begin{array}{lll}4 & \text { Discussion } & 16\end{array}$

$\begin{array}{ll}\text { A Eigenvalues \& eigenstates of the defect matrix } & 17\end{array}$

B Unitarity, crossing symmetry \& Casimir operators 18

$\begin{array}{ll}\text { C The XXZ model: generic values of the spin } & 20\end{array}$

\section{Introduction}

The presence of impurities in physical systems is an issue of great consequence, especially when dealing with more realistic models, and confronting experimental data. Along this spirit, integrability offers a framework where impurities may be naturally incorporated in a controllable manner. Although there have been numerous recent advances in both quantum [1]-[12], and classical [13]-[26] models with integrable defects, many questions still remain open. In the present investigation, we restrict our attention on quantum spin chains in the presence of a single integrable defect and extract the physical information concerning scattering processes within such models, directly from the Bethe ansatz equations. Our approach thus aims at complementing and enhancing the picture described in earlier works [5] in the context of integrable field theories.

The algebraic frame describing the presence of a point-like defect in a discrete integrable theory is by now well established through the quantum inverse scattering method (QISM) [27-31]. The formulation is based on the existence of a defect Lax operator that satisfies the same quadratic quantum algebra as the bulk monodromy matrix. In general, let us consider a one dimensional $(N+1)$-site theory with a point like defect on the $n$th site. In this case the modified monodromy matrix of the theory reads as

$$
T(\lambda)=L_{0 N+1}(\lambda) L_{0 N}(\lambda) \ldots \tilde{L}_{0 n}(\lambda-\Theta) \ldots L_{01}(\lambda)
$$


where $L$ corresponds to the "bulk" theory, $\tilde{L}$ corresponds to the defect and $\Theta$ is an arbitrary constant corresponding to the "rapidity" of the defect. Both Lax operators satisfy the same quadratic algebra

$$
R_{12}\left(\lambda_{1}-\lambda_{2}\right) L_{1}\left(\lambda_{1}\right) L_{2}\left(\lambda_{2}\right)=L_{2}\left(\lambda_{2}\right) L_{1}\left(\lambda_{1}\right) R_{12}\left(\lambda_{1}-\lambda_{2}\right),
$$

where the $R$-matrix is a solution of the Yang-Baxter equation (see e.g. [29-31] and references therein). The monodromy matrix of the theory $T(\lambda)$, naturally satisfies (1.2), guaranteeing the integrability of the model. The Hamiltonian of any generic system with a point-like defect is given by

$$
\mathcal{H} \propto-\left(\sum_{n, n-1 \neq j=1}^{N+1} \dot{\check{R}}_{j j+1}(0)+\dot{\tilde{L}}_{n+1 n}(0) \tilde{L}_{n+1 n}^{-1}(0)+\tilde{L}_{n+1 n}(0) \dot{\tilde{R}}_{n-1 n+1}(0) \tilde{L}_{n+1 n}^{-1}(0)\right)
$$

the "dot" denotes the derivative with respect to the spectral parameter. We focus here on the situation where $L(\lambda) \equiv R(\lambda)$, also define $\check{R}=\mathcal{P} R, \mathcal{P}$ is the permutation operator. Recall that the $R$ matrix reduces to the permutation operator at $\lambda=0$.

Note that here we are going to focus on the anti-ferromagnetic regime of the XXX and XXZ models. The derivation of the Bethe ansatz equations (BAE) is straightforward in the case where highest weight states exist. Amongst others, the thermodynamic limit of the BAE provides us with the scattering information for the given model, which we exploit in order to derive our results. Generalization of our results in the presence of multiple defects is straightforward within the QISM frame.

In the subsequent sections, we investigate the interaction between the particle like excitations displayed in the XXX and XXZ spin chains and the defect. These interactions are described by generic transmission matrices, that satisfy the quadratic algebra (1.2) with an overall physical factor, which is explicitly computed by means of the BAE. The XXZ model with a defect is studied in both the attractive and the repulsive regime. In the latter the formation of bound states between solitons and anti-solitons, called breathers, is allowed. After describing the scattering process for the breathers, we derive the transmission amplitude between a breather and the defect of the theory. We also compare our findings with earlier results, reaching complete agreement. Finally, the appendices contain several technical points and physical checks that further confirm our results.

\section{The isotropic case: $\mathrm{XXX}$ model}

We begin our analysis considering the isotropic XXX spin chain in the presence of a single defect (see also e.g. [32]-[36] and references therein). Let us first recall the quantum Lax operators for the bulk model, $L$, and the for the defect $\tilde{L}$. The generic defect matrix is given by

$$
\tilde{L}(\lambda)=\left(\begin{array}{cc}
\lambda+i S^{z}+\frac{i}{2} & i S^{-} \\
i S^{+} & \lambda-i S^{z}+\frac{i}{2}
\end{array}\right)
$$

where the algebraic objects $S^{z}, S^{ \pm}$are the generators of the $\mathfrak{s u}_{2}$ algebra,

$$
\left[S^{z}, S^{ \pm}\right]= \pm S^{ \pm}, \quad\left[S^{+}, S^{-}\right]=2 S^{z}
$$


The bulk $L$-matrix is actually the $R$-matrix of the model, which corresponds to the spin- $\frac{1}{2}$ representation of the $\mathfrak{s u}_{2}$, that is the following identifications are implemented in (2.1):

$$
S^{z} \mapsto \frac{\sigma^{z}}{2}, \quad S^{ \pm} \mapsto \sigma^{ \pm}
$$

As usual, $\sigma^{z}, \sigma^{ \pm}$denote the familiar $2 \times 2$ Pauli matrices. In the finite case, for the $\mathrm{n}=2 S+1$ dimensional representation of spin $S$, the algebraic objects $S^{z}$ and $S^{ \pm}$are represented by $\mathrm{n} \times \mathrm{n}$ matrices defined as

$$
S^{z}=\sum_{k=1}^{\mathrm{n}} \alpha_{k} e_{k k}, \quad S^{+}=\sum_{k=1}^{\mathrm{n}-1} C_{k} e_{k k+1}, \quad S^{-}=\sum_{k=1}^{\mathrm{n}-1} C_{k} e_{k+1 k}
$$

where we define the matrix elements: $\left(e_{i j}\right)_{k l}=\delta_{i k} \delta_{j l}$ and

$$
\alpha_{k}=\frac{1}{2}(\mathrm{n}+1-2 k), \quad C_{k}=\sqrt{k(\mathrm{n}-k)} .
$$

This choice may be thought of as the isotropic analogue of the type-II defect studied in [5], which will be analyzed in the subsequent sections within the XXZ spin chain context. The findings of this section may be seen as the quantum discrete analogues of the results on the Landau-Lifshitz model [26]. Classical scattering in the context of the LandauLifshitz model should be considered and comparison with our findings should provide a more concrete correspondence between the classical and quantum models in the presence of defects. The Hamiltonian of the model is given by $(1.3)$, where $\dot{\tilde{L}}(0)=\mathbb{I}$,

$$
\begin{aligned}
& \dot{\check{R}}_{j j+1}(0)=\frac{1}{2}\left(\sigma_{j}^{x} \sigma_{j+1}^{x}+\sigma_{j}^{y} \sigma_{j+1}^{y}+\sigma_{j}^{z} \sigma_{j+1}^{z}+\mathbb{I}_{j} \mathbb{I}_{j+1}\right), \\
& \tilde{L}_{n+1 n}(0)=\frac{i}{2}\left(S_{n}^{x} \sigma_{n+1}^{x}+S_{n}^{y} \sigma_{n+1}^{y}+2 S_{n}^{z} \sigma_{n+1}^{z}+\mathbb{I}_{n} \mathbb{I}_{n+1}\right) .
\end{aligned}
$$

The generic defect matrix as well as the bulk $L$-matrix possess highest weight states $\left(S^{+}|+\rangle=0\right)$, thus the typical algebraic Bethe variation may be applied and the corresponding Bethe ansatz equations (BAE) are immediately obtained ${ }^{1}$

$$
e_{y}\left(\lambda_{i}-\Theta\right) e_{1}^{N}\left(\lambda_{i}\right)=-\prod_{j=1}^{N} e_{2}\left(\lambda_{i}-\lambda_{j}\right), \quad y=2 S,
$$

where $\Theta$ is the rapidity associated to the defect, and we also define:

$$
e_{k}(\lambda)=\frac{\lambda+\frac{i k}{2}}{\lambda-\frac{i k}{2}}
$$

Having the Bethe ansatz equations at our disposal, we are now in a position to derive the physical quantities which describe the scattering processes on the chain.

\footnotetext{
${ }^{1}$ The BAE are valid for any $S \neq 0$ real number.
} 


\subsection{The transmission matrix}

Before we proceed with our analysis we shall recall that in the thermodynamic limit the solutions of the BAE may be expressed as "strings" with a real and an imaginary part. This is based on the so called "string hypothesis", stating that the Bethe roots may be cast as

$$
\lambda^{(n, j)}=\lambda_{0}+\frac{i}{2}(n+1-2 j), \quad j \in\{1, \ldots, n\} .
$$

Recall also that the total spin of a state may be obtained through the asymptotic behavior of the transfer matrix, and is given by the following familiar expression:

$$
S^{z}=\frac{N}{2}+S-M
$$

The spin $S^{z}$ of the state should be non-negative, thus the restriction $M \leq \frac{N}{2}+S$ is manifest (below the "equator"), while the rest of the states can be obtained by starting from a reference state, which is a "lowest weight" state $\left(S^{-}|-\rangle=0\right)$. The energy and momentum may be also explicitly expressed in terms of the Bethe roots $\left\{\lambda_{j}\right\}$ (for more details on the Bethe ansatz formulation and relevant physical expressions see also e.g. [27, 28, 37, 38]).

As is well known, the anti-ferromagnetic ground state is a "filled Fermi sea" with real solutions, i.e. 1-string configurations. It is easy to check that the ground state has an overall non zero spin $S^{z}=\tilde{S}$, where $\tilde{S} \equiv S-\frac{1}{2}$ is the "shifted" spin, which naturally emerges through the Bethe ansatz approach, as will be transparent subsequently when deriving the transmission matrix. Implementation of suitable string configurations can "correct" the spin, i.e. provide a total spin zero, without modifying the state's energy. In general, it is straightforward to show that in the presence of an $n$-string the spin becomes

$$
S^{z}=\tilde{S}-(n-1),
$$

while the energy and the momentum of the configuration are left intact.

A generic state contains $m$ particle-like excitations, which are interpreted as $m$ holes in the "filled Fermi sea". The density of the state may be obtained then, here we follow the standard formulation $[27,28,39]$ for the derivation. Starting from the Bethe ansatz equations, and after taking the logarithm and differentiating, we define the density of the state in the presence of $m$ holes in the filled Fermi sea as:

$$
\sigma(\lambda)=a_{1}(\lambda)+\frac{1}{N} a_{y}(\lambda-\Theta)-\int_{-\infty}^{\infty} d \lambda^{\prime} a_{2}\left(\lambda-\lambda^{\prime}\right) \sigma\left(\lambda^{\prime}\right)+\frac{1}{N} \sum_{j=1}^{m} a_{2}\left(\lambda-\tilde{\lambda}_{j}\right),
$$

where we have defined

$$
a_{n}(\lambda)=\frac{i}{2 \pi} \frac{d}{d \lambda} \ln \left(e_{n}(\lambda)\right)
$$

Passing to the thermodynamic limit, we have exploited the following basic formula in the presence of $m$ holes, with associated rapidities $\tilde{\lambda}_{j}$ :

$$
\frac{1}{N} \sum_{j=1}^{M} f\left(\lambda_{j}\right) \rightarrow \int_{-\infty}^{\infty} d \lambda f(\lambda) \sigma(\lambda)-\frac{1}{N} \sum_{j=1}^{m} f\left(\tilde{\lambda}_{j}\right)
$$


The Fourier transform of $a_{n}$ is needed subsequently, and is given by ${ }^{2}$

$$
\hat{a}_{n}(\omega)=e^{-n \frac{|\omega|}{2}} .
$$

For our purposes here, we shall focus on the case where $m=2(N$ is assumed to be odd), in order to derive the "kink" scattering matrix, as well as the transmission matrix. Using the machinery described above, the density may be expressed in a compact form as

$$
\sigma(\lambda)=\sigma_{0}(\lambda)+\frac{1}{N}\left(\sum_{k=1}^{2} r_{s}\left(\lambda-\tilde{\lambda}_{k}\right)+r_{t}(\lambda-\Theta)\right),
$$

with $\sigma_{0}(\lambda)$ being the density of the ground state. The Fourier transforms of the latter quantities have been computed

$$
\hat{\sigma}_{0}(\omega)=\frac{1}{2 \cosh \left(\frac{\omega}{2}\right)}, \quad \hat{r}_{s}(\omega)=\frac{e^{-\frac{|\omega|}{2}}}{2 \cosh \left(\frac{\omega}{2}\right)}, \quad \hat{r}_{t}(\omega)=\frac{e^{-(y-1) \frac{|\omega|}{2}}}{2 \cosh \left(\frac{\omega}{2}\right)} .
$$

Recall also that

$$
\sigma_{0}(\lambda)=\varepsilon(\lambda), \quad \text { and } \quad \varepsilon(\lambda)=\frac{1}{2 \pi} \frac{d p(\lambda)}{d \lambda},
$$

with $\varepsilon$ and $p$ being the energy and the momentum of the hole excitation (kink), respectively. Recall also that

$$
\sigma(\lambda)=\frac{1}{N} \frac{d h(\lambda)}{d \lambda}
$$

$h(\lambda)$ is the so-called counting function and $h\left(\tilde{\lambda}_{i}\right)=J_{i}$, where $J_{i}$ are integer numbers.

In order to identify the scattering amplitude between two holes as well as the hole-defect transmission amplitude, we compare the expression providing the density of the state (2.17) with the so called quantization condition, with respect to the hole with rapidity $\tilde{\lambda}_{1}$ :

$$
\left(e^{i N p\left(\tilde{\lambda}_{1}\right)} S\left(\tilde{\lambda}_{1}, \tilde{\lambda}_{2}, \Theta\right)-1\right)\left|\tilde{\lambda}_{1}, \tilde{\lambda}_{2}\right\rangle=0,
$$

where $S=e^{i \Phi}$, and $p\left(\tilde{\lambda}_{1}\right)$ is the momentum of the respective hole. Comparison of the quantization condition with the state's density (2.17) would provide the "kink-kink" scattering amplitude as well as the transmission amplitude, given that the factorization of the scattering is evident (see also [40]). More precisely, the study of the one-hole state would simply provide the transmission amplitude, which physically describes the interaction between the particle-like excitation and the defect, thus factorization of the type: $S\left(\tilde{\lambda}_{1}, \tilde{\lambda}_{2}, \Theta\right)=S_{s}\left(\tilde{\lambda}_{1}, \tilde{\lambda}_{2}\right) T\left(\tilde{\lambda}_{1}, \Theta\right)$, in the case of the two-hole state is manifest. Keeping these considerations in mind, the kink-kink amplitude as well as the transmission amplitude for the XXX model with a single defect can be derived then as

$$
S_{s}(\lambda)=\exp \left[-\int_{-\infty}^{\infty} \frac{d \omega}{\omega} e^{-i \omega \lambda} \hat{r}_{s}(\omega)\right], \quad T(\hat{\lambda})=\exp \left[-\int_{-\infty}^{\infty} \frac{d \omega}{\omega} e^{-i \omega \hat{\lambda}} \hat{r}_{t}(\omega)\right],
$$

where $\lambda \equiv \tilde{\lambda}_{1}-\tilde{\lambda}_{2}$ and $\hat{\lambda} \equiv \tilde{\lambda}_{1}-\Theta$.

\footnotetext{
${ }^{2}$ We have considered the following conventions for the Fourier transformations:

$$
f(\lambda)=\frac{1}{2 \pi} \int d \omega e^{-i \omega \lambda} \hat{f}(\omega), \quad \hat{f}(\omega)=\int d \lambda e^{i \omega \lambda} f(\lambda) .
$$
}


Taking now into account the following useful identity

$$
\frac{1}{2} \int_{0}^{\infty} \frac{d \omega}{\omega} \frac{e^{-\frac{\mu \omega}{2}}}{\cosh \frac{\omega}{2}}=\ln \frac{\Gamma\left(\frac{\mu+1}{4}\right)}{\Gamma\left(\frac{\mu+3}{4}\right)},
$$

the familiar expression for the XXX kink-kink scattering amplitude $[27,28,41]$ is reproduced in terms of $\Gamma$-functions

$$
S_{s}(\lambda)=\frac{\Gamma\left(-\frac{i \lambda}{2}+\frac{1}{2}\right) \Gamma\left(\frac{i \lambda}{2}+1\right)}{\Gamma\left(-\frac{i \lambda}{2}+1\right) \Gamma\left(\frac{i \lambda}{2}+\frac{1}{2}\right)} .
$$

This is the first eigenvalue of the scattering matrix and it is three-fold degenerate due to the underlying $\mathfrak{s u}_{2}$ symmetry. There is one more eigenvalue corresponding to the singlet state, which may be derived by considering the state with two holes and one 2-string with a real center:

$$
\lambda_{0}=\frac{\tilde{\lambda}_{1}+\tilde{\lambda}_{2}}{2}
$$

The second eigenvalue may be identified then, and turns out to be

$$
\frac{S_{2}(\lambda)}{S_{s}(\lambda)}=\frac{i \lambda-1}{i \lambda+1} .
$$

The $S$-matrix, which satisfies the Yang-Baxter equation, may be then cast as:

$$
\mathbb{S}(\lambda)=\frac{S_{s}(\lambda)}{i \lambda+1}\left(\begin{array}{cccc}
i \lambda+1 & & & \\
& i \lambda & 1 & \\
& 1 & i \lambda & \\
& & & i \lambda+1
\end{array}\right)
$$

which is the familiar XXX scattering matrix [27, 28]. Unitarity and crossing are also explicitly checked and are satisfied by the extracted $S$-matrix (see appendix A).

The transmission factor may be also identified through expression (2.22), and is found to be given by

$$
T(\hat{\lambda}, \tilde{S})=\frac{\Gamma\left(\frac{i \hat{\lambda}}{2}+\frac{\tilde{S}}{2}+\frac{3}{4}\right) \Gamma\left(-\frac{i \hat{\lambda}}{2}+\frac{\tilde{S}}{2}+\frac{1}{4}\right)}{\Gamma\left(\frac{i \hat{\lambda}}{2}+\frac{\tilde{S}}{2}+\frac{1}{4}\right) \Gamma\left(-\frac{i \hat{\lambda}}{2}+\frac{\tilde{S}}{2}+\frac{3}{4}\right)} .
$$

The spin associated to this state is identified through the BAE, and is given as: $S^{z}=$ $\tilde{S}+\frac{1}{2},\left(\tilde{S}=S-\frac{1}{2}\right)$, corresponding to the highest spin $S^{z}$ eigenvalue (appendix A). Notice the "shift" of the spin which naturally emerges through the Bethe ansatz process. We have been able so far to identify the first eigenvalue of the transmission matrix. As shown in the appendix A, there are only two distinct $(\tilde{\mathrm{n}}=2 \tilde{S}+1)$-fold degenerate eigenvalues associated to the generic transmission matrix.

As already pointed out, in order to identify the transmission amplitude, it is sufficient to consider the state with one hole ( $N$ even) (see also e.g. [42]). The other distinct eigenvalue may be found using suitable string configurations that modify the spin, but leave the energy of the sate intact. Considering $n$-strings, suitably positioned with respect to $\tilde{\lambda}_{1}$ and $\Theta$, one may determine each eigenvalue corresponding to the appropriate spin $S^{z}$ eigenvalue 
$S^{z} \in\left\{S_{\text {min }}^{z}, \ldots, \tilde{S}+1 / 2\right\}$. The rest of the negative spins may be obtained starting from a reference state, which is a "lowest" weight state (see also appendix A). For instance, it is easy to identify the spin $S^{z}$ eigenvalue of a state with one hole of rapidity $\tilde{\lambda}_{1}$, and one $n$-string: $n \in\left\{2, \ldots, n_{0}\right\}$, where we define

$$
\begin{array}{ll}
n_{0}=\tilde{S}+\frac{3}{2} \Rightarrow S_{\min }^{z}=0, & \tilde{S} \text { half-integer } \\
n_{0}=\tilde{S}+1 \Rightarrow S_{\min }^{z}=\frac{1}{2}, & \tilde{S} \text { integer. }
\end{array}
$$

The spin turns out then to be

$$
S^{z}=\tilde{S}+\frac{1}{2}-(n-1)
$$

The real center of the $n$-string is positioned at

$$
\lambda_{0}=\frac{x-1}{x} \tilde{\lambda}_{1}+\frac{\Theta}{x}, \quad x=\frac{2 S}{n-1} .
$$

The position of the $n$-string is determined using suitable quantum numbers, which characterize the state. We shall not give the technical details of the proof here, however the interested reader is referred to [38] for more details. Given the arguments above, the second eigenvalue of the transmission matrix can be derived for such a state:

$$
\frac{T_{2}(\hat{\lambda}, \tilde{S})}{T(\hat{\lambda}, \tilde{S})}=\frac{i \hat{\lambda}-\tilde{S}-\frac{1}{2}}{i \hat{\lambda}+\tilde{S}+\frac{1}{2}}
$$

corresponding to the spin eigenvalue (2.30).

Having the eigenvalues of the transmission matrix at hand, and bearing in mind that for purely transmitting defects the following quadratic algebra is satisfied $[1,2]$

$$
\mathbb{S}_{12}\left(\lambda_{1}-\lambda_{2}\right) \mathbb{T}_{1}\left(\lambda_{1}\right) \mathbb{T}_{2}\left(\lambda_{2}\right)=\mathbb{T}_{2}\left(\lambda_{2}\right) \mathbb{T}_{1}\left(\lambda_{1}\right) \mathbb{S}_{12}\left(\lambda_{1}-\lambda_{2}\right)
$$

where $\mathbb{S}$ is given in $(2.27)$, we conclude that

$$
\mathbb{T}(\hat{\lambda}, \tilde{S})=\frac{T(\hat{\lambda}, \tilde{S})}{i \hat{\lambda}+\tilde{S}+\frac{1}{2}}\left(\begin{array}{cc}
i \hat{\lambda}+S^{z}+\frac{1}{2} & S^{-} \\
S^{+} & i \hat{\lambda}-S^{z}+\frac{1}{2}
\end{array}\right) .
$$

This is the generic matrix associated this time to the spin $\tilde{S}=S-\frac{1}{2}$ representation of $\mathfrak{s u}_{2}$. The "shift" of the spin via the Bethe ansatz process, is once more pointed out.

The findings of this paragraph can be further confirmed by checking the basic requirements of unitarity and crossing symmetry, and of course by recalling that the transmission matrix has to satisfy (2.33). The transmission matrix presented above clearly satisfies the fundamental algebraic relation due to its structure. Unitarity and crossing symmetry have been also checked by inspection and confirmed for the transmission matrix (2.34) (see appendix $\mathrm{B}$ for details). In addition to the derivation of the transmission matrix eigenvalues via the BAE, there exist strong physical and algebraic arguments, as well as various checks regarding the validity of (2.34) as is manifest from the discussion above. It is also worth noting that this is the first time to our knowledge that such expressions are computed in the context of the XXX spin chain with a defect from the Bethe ansatz equations, and expressions (2.28), (2.34) are as far as we can tell novel. 


\section{The anisotropic case: $\mathrm{XXZ}$ model}

Our investigation on the defects is carried on with the anisotropic XXZ spin chain. Based on the analysis of this model we shall be able to make contact with relevant results extracted in the context of the sine-Gordon model. We shall focus in the present study on the so called type-II defects [5]. Let us first introduce the general defect $\tilde{L}$-matrix [43]:

$$
\tilde{L}(\lambda)=\left(\begin{array}{cc}
\sinh \left(\mu\left(\lambda+i S^{z}+\frac{i}{2}\right)\right) & \sinh (i \mu) S^{-} \\
\sinh (i \mu) S^{+} & \sinh \left(\mu\left(\lambda-i S^{z}+\frac{i}{2}\right)\right)
\end{array}\right),
$$

where we define $q=e^{i \mu}$, measuring the anisotropy of the model as $\Delta \equiv \cosh i \mu$. The bulk $L$-matrix is basically the $R$-matrix of the XXZ model, that is the spin $\frac{1}{2}$ representation of the latter expression, i.e. one simply implements in (3.1) the identifications (2.3).

The $\tilde{L}$-matrix is the typical solution of the quadratic algebra associated to $\mathfrak{U}_{q}\left(\mathfrak{s l}_{2}\right)$, which reads as:

$$
\left[S^{z}, S^{ \pm}\right]= \pm S^{ \pm}, \quad\left[S^{+}, S^{-}\right]=\frac{q^{2 S^{z}}-q^{-2 S^{z}}}{q-q^{-1}}
$$

In the finite case, which will be considered here, the generators are represented by $\mathrm{n} \times \mathrm{n}$ $(\mathrm{n}=2 S+1)$, matrices as:

$$
S^{z}=\sum_{k=1}^{\mathrm{n}} \alpha_{k} e_{k k}, \quad S^{+}=\sum_{k=1}^{\mathrm{n}-1} \tilde{C}_{k} e_{k k+1}, \quad S^{-}=\sum_{k=1}^{\mathrm{n}-1} \tilde{C}_{k} e_{k+1 k},
$$

where we define

$$
\alpha_{k}=\frac{1}{2}(\mathrm{n}+1-2 k), \quad \tilde{C}_{k}=\sqrt{[k]_{q}[\mathrm{n}-k]_{q}}, \quad[x]_{q}=\frac{q^{x}-q^{-x}}{q-q^{-1}} .
$$

Note that in [5] the $\tilde{L}$-matrix (3.1) is also used, but an infinite dimensional representation is employed; nevertheless, the structure of the defect matrices as well as the extracted physical quantities presented in [5] are similar to our expressions, as will be evident later in the text. The Hamiltonian of the model is given by $(1.3)$, where $\dot{\tilde{L}}(0)=\mu \operatorname{diag}\left(\cosh i \mu\left(S^{z}+\right.\right.$ $\left.\left.\frac{1}{2}\right), \cosh i \mu\left(-S^{z}+\frac{1}{2}\right)\right)$,

$$
\begin{aligned}
\dot{\check{R}}_{j j+1}(0)= & \frac{\mu}{2}\left(\sigma_{j}^{x} \sigma_{j+1}^{x}+\sigma_{j}^{y} \sigma_{j+1}^{y}+\cosh (i \mu) \sigma_{j}^{z} \sigma_{j+1}^{z}+\cosh (i \mu) \mathbb{I}_{j} \mathbb{I}_{j+1}\right) \\
\tilde{L}_{n+1 n}(0)= & \frac{\sinh (i \mu)}{2}\left(S_{n}^{x} \sigma_{n+1}^{x}+S_{n}^{y} \sigma_{n+1}^{y}\right) \\
& +\cosh \left(\frac{i \mu}{2}\right) \sinh \left(i \mu S_{n}^{z}\right) \sigma_{n+1}^{z}+\sinh \left(\frac{i \mu}{2}\right) \cosh \left(i \mu S_{n}^{z}\right) \mathbb{I}_{n+1} .
\end{aligned}
$$

The generic defect matrix and the bulk $L$-matrix possess highest weight states, thus as in the isotropic case the typical algebraic Bethe variation may be applied and the corresponding Bethe ansatz equations are immediately obtained ${ }^{3}$ having the following standard form

$$
e_{y}\left(\lambda_{i}-\Theta\right) e_{1}^{N}\left(\lambda_{i}\right)=-\prod_{j=1}^{N} e_{2}\left(\lambda_{i}-\lambda_{j}\right), \quad y=2 S,
$$

\footnotetext{
${ }^{3}$ The BAE are valid for any $S \neq 0$ real number.
} 
with $\Theta$ being again the rapidity of the defect, and we also introduce the notation

$$
e_{n}(\lambda)=\frac{\sinh \left(\mu\left(\lambda+\frac{i n}{2}\right)\right)}{\sinh \left(\mu\left(\lambda-\frac{i n}{2}\right)\right)}
$$

We may now proceed with the derivation of the transmission matrix distinguishing two regimes, the repulsive and the attractive, depending on the value of the coupling constant. Bear in mind that we wish to compare our findings with similar results in the context of the sine-Gordon model. Therefore, it is useful to provide the relation between the sine-Gordon coupling constant $\beta^{2}$, and the anisotropy parameter $\mu$ of the XXZ model (see also e.g. [44] for a more detailed discussion):

$$
\begin{aligned}
& \beta^{2}=8(\pi-\mu), \quad 4 \pi<\beta^{2}<8 \pi \quad \text { repulsive regime, } \\
& \beta^{2}=8 \mu, \quad 0<\beta^{2}<4 \pi \quad \text { attractive regime. }
\end{aligned}
$$

Note that in the attractive regime the formulation of bounds states between solitons and anti-solitons of zero spin (topological charge), the so called "breathers" is allowed.

\subsection{The repulsive regime; the transmission matrix}

We shall first consider the repulsive regime, and derive the corresponding transmission matrix. In this regime the antiferromagnetic ground state is a filled Fermi sea with real strings ( $N$ odd), as in the isotropic case. For our purposes, it suffices to consider here the state with two holes. The density associated to this state is obtained in the thermodynamic limit by following the logic described in the previous section

$$
\sigma(\lambda)=a_{1}(\lambda)+\frac{1}{N} a_{y}(\lambda-\Theta)-\int_{-\infty}^{\infty} d \lambda^{\prime} a_{2}\left(\lambda-\lambda^{\prime}\right) \sigma\left(\lambda^{\prime}\right)+\frac{1}{N} \sum_{j=1}^{2} a_{2}\left(\lambda-\tilde{\lambda}_{j}\right)
$$

where the basic formula in the presence of two "holes" of rapidities $\tilde{\lambda}_{j}(2.14)$ was exploited. Note that $a_{n}(\lambda)$ is defined again as in (2.13), whereas its Fourier transformation is given by

$$
\hat{a}_{n}(\omega)=\frac{\sinh \left((\nu-n) \frac{\omega}{2}\right)}{\sinh \left(\frac{\nu \omega}{2}\right)}, \quad \nu=\frac{\pi}{\mu}, \quad 0<n<2 \nu .
$$

In the present subsection, we restrict ourselves to the values $0<y<2 \nu$. Results on generic values of $y$ are presented in appendix $\mathrm{C}$.

As in the isotropic case, the density may be expressed in a compact form as

$$
\sigma(\lambda)=\sigma_{0}(\lambda)+\frac{1}{N}\left(\sum_{k=1}^{2} r_{s}\left(\lambda-\tilde{\lambda}_{k}\right)+r_{t}(\lambda-\Theta)\right)
$$

The Fourier transforms of the latter quantities have been explicitly determined

$$
\begin{aligned}
& \hat{\sigma}_{0}(\omega)=\frac{1}{2 \cosh \left(\frac{\omega}{2}\right)}, \quad \hat{r}_{s}(\omega)=\frac{\sinh \left((\nu-2) \frac{\omega}{2}\right)}{2 \sinh \left((\nu-1) \frac{\omega}{2}\right) \cosh \left(\frac{\omega}{2}\right)}, \\
& \hat{r}_{t}(\omega)=\frac{\sinh \left((\nu-y) \frac{\omega}{2}\right)}{2 \sinh \left((\nu-1) \frac{\omega}{2}\right) \cosh \left(\frac{\omega}{2}\right)} .
\end{aligned}
$$


Relations (2.19) for the energy and momentum of the particle-like ("soliton") excitation are also valid here.

In order to identify the scattering amplitude between two holes, we compare the expression providing the density of the state with the quantization condition (2.21), with respect to the excitation of rapidity $\tilde{\lambda}_{1}$. We are now in the position to compute the soliton-soliton scattering amplitude, as well as the transmission amplitude for the XXZ model using the expressions (2.22). Taking into account the following useful identity

$$
\frac{1}{4} \int_{0}^{\infty} \frac{d x}{x} \frac{e^{-\mu x}}{\sinh x \sinh \beta x}=\ln \prod_{k=0}^{\infty} \Gamma\left(\frac{\mu}{2}+\frac{\beta}{2}+k \beta+\frac{1}{2}\right),
$$

we may easily reproduce the well known expression for the sine-Gordon soliton-soliton scattering (see also $[5,45]$ ),

$$
\begin{aligned}
S_{s}(\lambda, \gamma)=\prod_{k=0}^{\infty} \frac{\Gamma(z+2(k+1) \gamma) \Gamma(z+2 k \gamma+1)}{\Gamma(z+(2 k+1) \gamma) \Gamma(z+(2 k+1) \gamma+1)} \\
\quad \times \frac{\Gamma(-z+(2 k+1) \gamma) \Gamma(-z+(2 k+1) \gamma+1)}{\Gamma(-z+2(k+1) \gamma) \Gamma(-z+2 k \gamma+1)},
\end{aligned}
$$

where we now define

$$
z=i \gamma \lambda, \quad \gamma=\frac{1}{\nu-1} .
$$

Suitable configurations corresponding to soliton/anti-soliton states may be formulated, so that all the $S$-matrix eigenvalues may be identified. We shall not give the details of such a derivation here, however we refer the interested reader to e.g. [44, 46] and references therein for a more detailed analysis. The $S$-matrix, solution of the Yang-Baxter equation, is structurally similar to the "bare" $R$-matrix, and is given by

$$
\mathbb{S}(\lambda)=\frac{S_{s}(\lambda, \gamma)}{a(\lambda, \gamma)}\left(\begin{array}{cccc}
a(\lambda, \gamma) & & & \\
& b(\lambda, \gamma) & c(\gamma) & \\
& c(\gamma) & b(\lambda, \gamma) & \\
& & & a(\lambda, \gamma)
\end{array}\right)
$$

where

$$
a(\lambda, \gamma)=\sin (\pi \gamma(i \lambda+1)), \quad \beta(\lambda, \gamma)=\sin (i \pi \gamma \lambda), \quad c(\gamma)=\sin (\pi \gamma) .
$$

Note that the $S$-matrix is essentially a renormalized $R$-matrix, given that both the spectral parameter as well as the anisotropy parameter are renormalized, as is clear from the expressions above. The $S$-matrix is in agreement with the results on the sine-Gordon model (see also $[5,44-46]$ ).

Recalling expressions (2.22) we can also extract the transmission amplitude. Based on the state described above, as well as the factorization argument, we find the first eigenvalue of the transmission matrix

$$
\begin{aligned}
T(\hat{\lambda}, \gamma, \tilde{S})=\prod_{k=0}^{\infty} & \frac{\Gamma\left(\hat{z}+\gamma \tilde{S}+\frac{\gamma}{2}+(2 k+1) \gamma\right) \Gamma\left(\hat{z}-\gamma \tilde{S}-\frac{\gamma}{2}+(2 k+1) \gamma+1\right)}{\Gamma\left(\hat{z}+\gamma \tilde{S}+\frac{\gamma}{2}+2 k \gamma\right) \Gamma\left(\hat{z}-\gamma \tilde{S}-\frac{\gamma}{2}+2(k+1) \gamma+1\right)} \\
& \times \frac{\Gamma\left(-\hat{z}+\gamma \tilde{S}+\frac{\gamma}{2}+2 k \gamma\right) \Gamma\left(-\hat{z}-\gamma \tilde{S}-\frac{\gamma}{2}+2(k+1) \gamma+1\right)}{\Gamma\left(-\hat{z}+\gamma \tilde{S}+\frac{\gamma}{2}+(2 k+1) \gamma\right) \Gamma\left(-\hat{z}-\gamma \tilde{S}-\frac{\gamma}{2}+(2 k+1) \gamma+1\right)},
\end{aligned}
$$


where $\hat{z}=i \hat{\lambda} \gamma$ and $\tilde{S}=S-\frac{1}{2}$ is the "shifted" spin. As already mentioned, in order to derive the transmission amplitude it is sufficient to consider the state with one hole. In this case, the corresponding spin eigenvalue is computed explicitly and turns out to be

$$
S^{z}=\frac{\nu}{\nu-1}\left(\tilde{S}+\frac{1}{2}\right) .
$$

One immediately observes an overall renormalization factor $\frac{\nu}{\nu-1}$, which is equal to the ratio of the bare anisotropy parameter $\nu$ over the renormalized one $\nu-1$; the physical (renormalized) spin reduces then to the expected one: $S_{p h .}^{z}=\tilde{S}+\frac{1}{2}$.

Alongside the $S$-matrix (3.15), the transmission matrix $\mathbb{T}$ satisfies the quadratic algebra (2.33). We conclude that the transmission matrix may be cast then as

$$
\mathbb{T}(\hat{\lambda}, \gamma, \tilde{S})=\frac{T(\hat{\lambda}, \gamma, \tilde{S})}{\sin \left(\pi \gamma\left(i \hat{\lambda}+\tilde{S}+\frac{1}{2}\right)\right)}\left(\begin{array}{cc}
\sin \left(\pi \gamma\left(i \hat{\lambda}+S^{z}+\frac{1}{2}\right)\right) & \sin (\pi \gamma) S^{-} \\
\sin (\pi \gamma) S^{+} & \sin \left(\pi \gamma\left(i \hat{\lambda}-S^{z}+\frac{1}{2}\right)\right)
\end{array}\right)
$$

where $q^{S^{z}}, S^{ \pm}$correspond to the spin $\tilde{S}$ representation of $\mathfrak{U}_{q}\left(\mathfrak{s l}_{2}\right)$, where now $q=e^{i \pi \gamma}$. We should point out that the transmission matrix is essentially a renormalized defect matrix, as is manifest from the structure of $\mathbb{T}$ and $\tilde{L}$-matrices. Having said this, it is straightforward to verify that $\mathbb{T}$ is indeed a solution of the fundamental algebra (2.33). Moreover, based on the analysis presented in appendix A, we have checked by inspection that the transmission matrix satisfies unitarity and crossing symmetry, hence its validity is completely confirmed. As in the isotropic case, appropriate string configurations suitably positioned with respect to $\tilde{\lambda}_{1}, \Theta$ provide the various eigenvalues of the transmission matrix. However, in the trigonometric case this is a highly intricate task, and will be left for separate investigations. It is also worth noting that this is the first time as far as we know that such expressions are computed via the Bethe ansatz formulation in the trigonometric case. A detailed comparison with earlier results on the transmission matrix of the sine-Gordon model will be given in the next subsection.

\subsection{The attractive regime}

\subsubsection{The soliton transmission matrix}

We shall now focus on the attractive regime; in this regime bound states between solitons and anti-solitons, the so-called "breathers", exist. Thus the scattering between the breathers and the defect may be also investigated. As was shown in earlier studies, the ground state in the attractive regime consists of the so-called negative parity strings (see also e.g. [44] and references therein)

$$
\lambda^{(-)}=\lambda+\frac{i \pi}{2 \mu} .
$$

The BAE are modified then as follows

$$
g_{y}\left(\lambda_{i}-\Theta\right) g_{1}^{N}(\lambda)=-\prod_{j=1}^{M} e_{2}\left(\lambda_{i}-\lambda_{j}\right)
$$


where we define

$$
g_{n}(\lambda)=\frac{\cosh \left(\mu\left(\lambda+\frac{i n}{2}\right)\right)}{\cosh \left(\mu\left(\lambda-\frac{i n}{2}\right)\right)}
$$

A generic state with two particle excitations (two holes in the filled Fermi sea of negative parity strings) is considered and the density associated to this state may be derived based on the standard formulation $[27,28,39]$. It turns out that the derived state density is given by the following expression

$$
-\sigma(\lambda)=b_{1}(\lambda)+\frac{1}{N} b_{y}(\lambda-\Theta)-\int_{-\infty}^{\infty} d \lambda^{\prime} a_{2}\left(\lambda-\lambda^{\prime}\right) \sigma\left(\lambda^{\prime}\right)+\frac{1}{N} \sum_{j=1}^{2} a_{2}\left(\lambda-\tilde{\lambda}_{j}\right),
$$

where the formula (2.14) in the presence of two "holes" of rapidities $\tilde{\lambda}_{j}$ has been exploited. The Fourier transformation of $a_{n}$ is given in (3.9), whereas the Fourier transform for $b_{n}$ is found to be

$$
\begin{array}{ll}
\hat{b}_{n}(\omega)=-\frac{\sinh \left(\frac{n \omega}{2}\right)}{\sinh \left(\frac{\nu \omega}{2}\right)}, & 0<n<\nu, \\
\hat{b}_{n}(\omega)=-\frac{\sinh \left((n-2 \nu) \frac{\omega}{2}\right)}{\sinh \left(\frac{\nu \omega}{2}\right)}, & \nu<n<2 \nu .
\end{array}
$$

For the sake of simplicity, we consider here the case $0<y<\nu$. Results regarding generic values of $y$ are presented in appendix C. Similarly to the previous sections, the density is compactly expressed as

$$
\sigma(\lambda)=\sigma_{0}(\lambda)+\frac{1}{N}\left(\sum_{k=1}^{2} r_{s}\left(\lambda-\tilde{\lambda}_{k}\right)+r_{t}(\lambda-\Theta)\right),
$$

whereas the Fourier transforms of the latter quantities are given by

$$
\begin{aligned}
& \hat{\sigma}_{0}(\omega)=\frac{1}{2 \cosh \left((\nu-1) \frac{\omega}{2}\right)}, \quad \hat{r}_{s}(\omega)=-\frac{\sinh \left((\nu-2) \frac{\omega}{2}\right)}{2 \sinh \left(\frac{\omega}{2}\right) \cosh \left((\nu-1) \frac{\omega}{2}\right)}, \\
& \hat{r}_{t}(\omega)=\frac{\sinh \left(y \frac{\omega}{2}\right)}{2 \sinh \left(\frac{\omega}{2}\right) \cosh \left((\nu-1) \frac{\omega}{2}\right)} .
\end{aligned}
$$

Note also that relations (2.19) also hold for the energy and momentum of the particlelike excitation.

Comparison of the expression providing the density of the state (3.25) with the quantization condition $(2.21)$, with respect to the excitation of rapidity $\tilde{\lambda}_{1}$, leads to the derivation of the soliton-soliton scattering as well as the transmission amplitude, given by (2.22). Taking into account the identity (3.12) we reproduce the well known expression for the sine-Gordon soliton-soliton scattering in the attractive regime (compare for instance with the notation used in [5]), which is given by (3.13), but now we define

$$
z=i \lambda, \quad \gamma=\nu-1
$$


Soliton anti-soliton configurations leading to all the $S$-matrix eigenvalues may be identified $[44,46]$. The $S$-matrix, solution of the Yang-Baxter equation as well, is structurally similar to the "bare" $R$-matrix, and is given as (3.15), where we now define

$$
a(\lambda, \gamma)=\sin \pi(i \lambda+\gamma), \quad \beta(\lambda, \gamma)=\sin i \pi \lambda, \quad c(\gamma)=\sin \pi \gamma .
$$

In a similar fashion, we also identify the transmission matrix, which describes the interaction between the soliton and the defect. Based on the state described above, as well as the factorization argument, we extract the first eigenvalue of the transfer matrix, which is expressed as

$$
\begin{aligned}
T(\hat{\lambda}, \gamma)=\prod_{k=0}^{\infty} \frac{\Gamma\left(\hat{z}-\xi+2(k+1) \gamma+\frac{1}{2}\right) \Gamma\left(\hat{z}+\xi+2 k \gamma+\frac{1}{2}\right)}{\Gamma\left(\hat{z}-\xi+(2 k+1) \gamma+\frac{1}{2}\right) \Gamma\left(\hat{z}+\xi+(2 k+1) \gamma+\frac{1}{2}\right)} \\
\times \frac{\Gamma\left(-\hat{z}-\xi+(2 k+1) \gamma+\frac{1}{2}\right) \Gamma\left(-\hat{z}+\xi+(2 k+1) \gamma+\frac{1}{2}\right)}{\Gamma\left(-\hat{z}-\xi+2(k+1) \gamma+\frac{1}{2}\right) \Gamma\left(-\hat{z}+\xi+2 k \gamma+\frac{1}{2}\right)}
\end{aligned}
$$

where

$$
\hat{z}=i \hat{\lambda}, \quad \xi=S+\frac{\gamma}{2} .
$$

The transmission matrix $\mathbb{T}$ satisfies the quadratic algebra (2.33) together with the $S$ matrix derived above. We conclude that the transmission matrix $\mathbb{T}$ may be then cast as $\left(\right.$ set $\left.\frac{i \hat{\lambda}}{\gamma}=i u\right)$

$$
\mathbb{T}(u, \gamma, \tilde{S})=\frac{T(u, \gamma, \tilde{S})}{\sin \left(\pi \gamma\left(i u+\tilde{S}+\frac{1}{2}\right)\right)}\left(\begin{array}{cc}
\sin \left(\pi \gamma\left(i u+S^{z}+\frac{1}{2}\right)\right) & \sin (\pi \gamma) S^{-} \\
\sin (\pi \gamma) S^{+} & \sin \left(\pi \gamma\left(i u-S^{z}+\frac{1}{2}\right)\right)
\end{array}\right) .
$$

The elements $S^{z}, S^{ \pm}$form now the spin $\tilde{S}=0$ representation of $\mathfrak{U}_{q}\left(\mathfrak{s l}_{2}\right)$ with $q^{i \pi \gamma}$, which is an infinite dimensional representation. Notice that here we have used the fact that: $\sin \left(\pi \gamma\left(i u+\frac{1}{2}-\frac{S+\frac{1}{2}}{\gamma}\right)\right)= \pm \sin \left(\pi \gamma\left(i u+\frac{1}{2}\right)\right)$ or $\pm \cos \left(\pi \gamma\left(i u+\frac{1}{2}\right)\right)$ given that $S+\frac{1}{2}$ is an integer or half-integer. Computation of the spin of the state with one hole via the Bethe ansatz equations leads to $S^{z}=\frac{\nu}{2}$, i.e. the renormalized spin is $\frac{1}{2}(\nu$ is the renormalization factor in the attractive regime), which is basically the spin of the hole, denoting that the defect spin is effectively zero, confirming the relevant result $(\tilde{S}=0)$ through the derivation of the transmission matrix.

To efficiently compare with earlier relevant results from the Sine-Gordon model [5], it is convenient to introduce some useful notation; first we shift $\lambda$ such that

$$
i \hat{\lambda} \rightarrow i \hat{\lambda}+\Lambda
$$

where $\Lambda$ is an arbitrary constant. Also define the following quantities:

$$
z_{j}=-i \hat{\lambda}-\frac{i \gamma}{\pi} \eta_{j}, \quad \eta_{1}=\frac{i \pi}{\gamma}(\Lambda+\xi), \quad \eta_{2}=\frac{i \pi}{\gamma}(\Lambda-\xi), \quad j \in\{1,2\} .
$$

The transmission factor is then expressed in terms of $z_{1}, z_{2}$ as

$$
T\left(z_{1}, z_{2}\right)=\frac{\sin \pi\left(z_{2}+\frac{1}{2}\right)}{\pi} \rho_{d}\left(z_{1}, z_{2}\right),
$$


where the overall physical factor $\rho_{d}$ is given by

$$
\begin{aligned}
\rho_{d}\left(z_{1}, z_{2}\right)=\Gamma\left(\frac{1}{2}\right. & \left.-z_{1}\right) \Gamma\left(\frac{1}{2}-z_{2}\right) \prod_{k=1}^{\infty} \frac{\Gamma\left(z_{1}+(2 k-1) \gamma+\frac{1}{2}\right) \Gamma\left(z_{2}+(2 k-1) \gamma+\frac{1}{2}\right)}{\Gamma\left(z_{1}+2 k \gamma+\frac{1}{2}\right) \Gamma\left(z_{2}+2 k \gamma+\frac{1}{2}\right)} \\
& \times \frac{\Gamma\left(-z_{1}+2 k \gamma+\frac{1}{2}\right) \Gamma\left(-z_{2}+2 k \gamma+\frac{1}{2}\right)}{\Gamma\left(-z_{1}+(2 k-1) \gamma+\frac{1}{2}\right) \Gamma\left(-z_{2}+(2 k-1) \gamma+\frac{1}{2}\right)} .
\end{aligned}
$$

The latter expression is identical to the one extracted in [5] for the type-II defects, taken into account the identifications (3.33). Having determined the soliton transmission matrix we now proceed with the derivation of the breather transmission matrix.

\subsubsection{The breather transmission amplitude}

The breathers are in general identified within the Bethe ansatz frame by suitable string configurations. To fully describe this scattering process for the breathers it is necessary to take into consideration two sets of Bethe ansatz equations; the first set describes the negative parity one-strings, while the second one describes the breather itself. The second set of BAE is necessary in order to derive the energy and momentum of the breather, and also compare with the quantization condition with respect to the breather (for more details on breathers and their interactions we refer the interested breather to [44] and references therein).

A state with two light breathers with rapidities $\bar{\lambda}_{1}, \bar{\lambda}_{2}$ will be considered. We shall basically deal with the lightest breathers for simplicity; a generalization of the results concerning higher breathers is then straightforward [44], and is given at the end of the subsection. The lightest breather is described by one positive parity (real) string with rapidity $\bar{\lambda}_{i}$, then the BAE for the state with two breathers are expressed as:

$$
g_{y}\left(\lambda_{i}-\Theta\right) g_{1}^{N}\left(\lambda_{i}\right)=-\prod_{j=1}^{M} e_{2}\left(\lambda_{i}-\lambda_{j}\right) \prod_{j=1}^{2} g_{2}\left(\lambda_{i}-\bar{\lambda}_{j}\right) .
$$

There is a second set of BAE describing the breather with rapidity $\bar{\lambda}_{1}$

$$
e_{y}\left(\bar{\lambda}_{1}-\Theta\right) e_{1}^{N}\left(\bar{\lambda}_{1}\right)=-\prod_{j=1}^{M} g_{2}\left(\bar{\lambda}_{1}-\lambda_{j}\right) e_{2}\left(\bar{\lambda}_{1}-\bar{\lambda}_{2}\right) .
$$

As already mentioned the second set is necessary for our purposes here, given that it facilitates the computation of the energy and momentum of the breather as well as the formulation of the corresponding quantization condition.

From the first set of BAE (3.36) the following density regarding the negative parity strings arises,

$$
\sigma(\lambda)=\sigma_{0}(\lambda)+\frac{1}{N}\left(\mathrm{~B}(\lambda-\Theta)+\sum_{j=1}^{2} \mathrm{R}\left(\lambda-\tilde{\lambda}_{j}\right)\right),
$$

where we define the Fourier transforms of R, B as

$$
\hat{\mathrm{R}}(\omega)=-\frac{\cosh \left(\frac{\omega}{2}\right)}{\cosh \left((\nu-1) \frac{\omega}{2}\right)}, \quad \hat{\mathrm{B}}(\omega)=\frac{\sinh \left(\frac{y \omega}{2}\right)}{2 \sinh \left(\frac{\omega}{2}\right) \cosh \left((\nu-1) \frac{\omega}{2}\right)}
$$


The second set (3.37) leads to the density describing the breather

$$
\bar{\sigma}(\lambda)=\bar{\sigma}_{0}(\lambda)+\left(t_{b}(\lambda-\Theta)+\sum_{j=1}^{2} r_{b}\left(\lambda-\bar{\lambda}_{j}\right)\right)
$$

where the corresponding Fourier transforms read as

$$
\hat{\bar{\sigma}}_{0}(\omega)=\frac{\cosh \left((\nu-2) \frac{\omega}{2}\right)}{\cosh \left((\nu-1) \frac{\omega}{2}\right)}, \quad \hat{r}_{b}(\omega)=-\frac{\cosh \left((\nu-3) \frac{\omega}{2}\right)}{\cosh \left((\nu-1) \frac{\omega}{2}\right)}, \quad \hat{t}_{b}(\omega)=\frac{\cosh \left((\nu-y-1) \frac{\omega}{2}\right)}{\cosh \left((\nu-1) \frac{\omega}{2}\right)} .
$$

Moreover, it may be shown as in the soliton case that

$$
\bar{\sigma}_{0}(\lambda)=\bar{\varepsilon}(\lambda), \quad \bar{\varepsilon}(\lambda)=\frac{1}{2 \pi} \frac{d \bar{p}(\lambda)}{d \lambda}
$$

where $\bar{\varepsilon}$ and $\bar{p}$ are the energy and momentum of the lightest breather respectively.

Similarly, a quantization condition for the breather may be formulated

$$
\left(e^{i \bar{p}\left(\lambda_{1}\right) N} \bar{S}\left(\bar{\lambda}_{1}, \bar{\lambda}_{2}, \Theta\right)-1\right)\left|\bar{\lambda}_{1}, \bar{\lambda}_{2}\right\rangle=0,
$$

due to the factorization of the scattering process $\bar{S}\left(\bar{\lambda}_{1}, \bar{\lambda}_{2}, \Theta\right)=S_{b}^{(1,1)}\left(\bar{\lambda}_{1}, \bar{\lambda}_{2}\right) T_{b}^{(1)}\left(\bar{\lambda}_{1}, \Theta\right)$. Comparison of the latter formula with $\bar{\sigma}$ leads to the expressions for the breather scattering amplitude as well as the corresponding breather transmission amplitude:

$$
S_{b}^{(1,1)}(\lambda)=\exp \left[-\int_{-\infty}^{\infty} \frac{d \omega}{\omega} e^{-i \omega \lambda} \hat{r}_{b}(\omega)\right], \quad T_{b}^{(1)}(\hat{\lambda})=\exp \left[-\int_{-\infty}^{\infty} \frac{d \omega}{\omega} e^{-i \omega \hat{\lambda}} \hat{t}_{b}(\omega)\right]
$$

$\lambda=\bar{\lambda}_{1}-\bar{\lambda}_{2}, \hat{\lambda}=\bar{\lambda}_{1}-\Theta$.

Bearing in mind (3.41), (3.44) as well as the useful identity (2.23) we conclude that the breather scattering amplitude is given by the following hyperbolic ratios,

$$
S_{b}^{(1,1)}(\theta)=-\frac{\sinh \left(\frac{\theta}{2}-\frac{i \pi}{2 \gamma}\right) \sinh \left(\frac{\theta}{2}+\frac{i \pi}{2 \gamma}+\frac{i \pi}{2}\right)}{\sinh \left(\frac{\theta}{2}+\frac{i \pi}{2 \gamma}\right) \sinh \left(\frac{\theta}{2}-\frac{i \pi}{2 \gamma}-\frac{i \pi}{2}\right)}
$$

where we define: $\theta=\frac{\pi \lambda}{\gamma}$. The scattering amplitude coincides of course with the familiar sine-Gordon breather quantity, and for the lightest breather, which we consider here this corresponds to the scattering amplitude of the scalar sinh-Gordon field [5, 44, 45].

Similarly, through (3.44) the breather transmission amplitude may be derived as

$$
T_{b}^{(1)}(\hat{\theta})=-\frac{\sinh \left(\frac{\hat{\theta}-\eta_{1}}{2}-\frac{i \pi}{4}\right) \sinh \left(\frac{\hat{\theta}-\eta_{2}}{2}-\frac{i \pi}{4}\right)}{\sinh \left(\frac{\hat{\theta}-\eta_{1}}{2}+\frac{i \pi}{4}\right) \sinh \left(\frac{\hat{\theta}-\eta_{2}}{2}+\frac{i \pi}{4}\right)}
$$

$\hat{\theta}=\frac{\pi \hat{\lambda}}{\gamma}$, the constants $\eta_{i}$ have been defined in (3.33), and it is clear that our expression (3.46) for the breather transmission amplitude coincides with the one identified in [5].

The results on the scattering and transmission amplitudes may be generalized for higher $n$-breathers, which are represented by $n$-positive parity strings with real centers $\bar{\lambda}_{j}$. 
More precisely, it is straightforward to see (see e.g. [44]) that the scattering between two generic $n_{1}, n_{2}$ breathers may be expressed as:

$$
S_{b}^{\left(n_{1}, n_{2}\right)}(\lambda)=\prod_{l_{1}=1}^{n_{1}} \prod_{l_{2}=1}^{n_{2}} S_{b}^{(1,1)}\left(\lambda+\frac{i}{2}\left(n_{1}-n_{2}-2 l_{1}+2 l_{2}\right)\right),
$$

while the transmission amplitude of an $n$-breather is

$$
T_{b}^{(n)}(\hat{\lambda})=\prod_{l=1}^{n} T_{b}^{(1)}\left(\hat{\lambda}+\frac{i}{2}(n+1-2 l)\right) .
$$

The spin of the one $n$-breather state in the presence of the defect can be also evaluated, and turns out to be $S^{z}=0$. The spin of the breather is as expected zero, recall that in the attractive regime as pointed out in the previous subsection the "effective" defect spin is zero. With this we conclude our analysis on the transmission matrices for the kinks (solitons) and breathers in the (an)isotropic Heisenberg models.

\section{Discussion}

We have studied in the present article one-dimensional (an)isotropic Heisenberg chains in the presence of a single impurity. We have recalled the construction of the kink $S$-matrix and have produced the transmission amplitudes through the Bethe ansatz equations. We have also been able to derive the breather's transmission matrix in the attractive regime of the XXZ chain. Our findings in the attractive regime of the XXZ model coincide with earlier results obtained in the context of the sine-Gordon model [5], implying that the picture is consistent. Our findings in the XXX case as well as in the repulsive regime of the XXZ model are novel as far as we can tell and involve finite representations of the $\mathfrak{s l}_{2}$ and $\mathfrak{U}_{q}\left(\mathfrak{s l}_{2}\right)$. As a further check, comparison with relevant results at the classical level should be made, especially with studies in the Landau-Lifshitz [26], and sine-Gordon models [23].

Some comments on future directions are in order here. We have based our formulation here on finite representations of $\mathfrak{s l}_{2}, \mathfrak{U}_{q}\left(\mathfrak{s l}_{2}\right)$. More precisely, we have restricted our analysis on defect matrices, that correspond to finite representations, thus the algebraic Bethe ansatz formulation may be applied. The chosen representations possess highest weight states, and therefore the algebraic Bethe ansatz variation can be successfully applied. However, infinite dimensional representations of $\mathfrak{s l}_{2}, \mathfrak{U}_{q}\left(\mathfrak{s l}_{2}\right)$ for the defect matrix, can be also considered. In this case local gauge (Darboux) transformations in the spirit described in $[47,48]$ should be employed in order to extract the associated spectrum and BAE.

Moreover, there exist several open issues to be resolved; it is an intriguing task to determine the string configurations as well as the positions of the real centers of the $n$ strings within the XXZ model, in order to extract all the eigenvalues of the trigonometric transmission matrix from the BAE. A natural generalization would also be to extend our analysis in the case of higher rank (deformed) algebras. Finally, depending on the values of the coupling constant it is possible to consider the formation of bound states between the particle-like excitations and the defect. This analysis may be achieved via the investigation of the poles appearing in the overall physical factor of the transmission matrix. All the above are significant issues, which hopefully will be addressed in the near future. 


\section{A Eigenvalues \& eigenstates of the defect matrix}

In the present appendix we identify the eigenvalues and the corresponding eigenstates of the defect matrix. Note that the spin operator shares essentially the same set of eigenstates. We begin with writing the defect matrix as

$$
\begin{aligned}
\tilde{L}(\lambda)= & \sum_{m=1}^{\mathrm{n}}\left(\mathcal{A}_{m} e_{11}^{(2)} \otimes e_{m m}^{(\mathrm{n})}+\mathcal{D}_{m} e_{22}^{(2)} \otimes e_{m m}^{(\mathrm{n})}\right) \\
& +\sum_{m=1}^{\mathrm{n}-1}\left(\mathcal{B}_{m} e_{12}^{(2)} \otimes e_{m+1 m}^{(\mathrm{n})}+\mathcal{C}_{m} e_{21}^{(2)} \otimes e_{m m+1}^{(\mathrm{n})}\right)
\end{aligned}
$$

$\tilde{L}(\lambda) \in \operatorname{End}\left(\mathbb{C}^{2} \otimes \mathbb{C}^{\mathrm{n}}[\lambda]\right), \mathrm{n}=2 S+1$. We consider the following ansatz for the eigenstates:

$$
\begin{aligned}
& \left|\psi_{0}\right\rangle=\hat{e}_{1}^{(2)} \otimes \hat{e}_{1}^{(\mathrm{n})}, \quad\left|\psi_{\mathrm{n}}\right\rangle=\hat{e}_{\mathrm{n}}^{(2)} \otimes \hat{e}_{\mathrm{n}}^{(\mathrm{n})} \\
& \left|\psi_{k}\right\rangle=f_{k} \hat{e}_{1}^{(2)} \otimes \hat{e}_{k+1}^{(\mathrm{n})}+f_{k+1} \hat{e}_{2}^{(2)} \otimes \hat{e}_{k}^{(\mathrm{n})}, \quad k=1, \cdots, n-1,
\end{aligned}
$$

where $\hat{e}_{k}^{(d)}$ is a $d$-dimensional column vector with zero elements everywhere, and the unit at the $k$-th position.

Acting with $\tilde{L}$ on the vector, according to the rule $e_{a b}^{(d)} \hat{e}_{c}^{(d)}=\delta_{b c} \hat{e}_{a}^{(d)}$, we find

$$
\tilde{L}(\lambda)\left|\psi_{0}\right\rangle=\left(\lambda+\frac{i \mathrm{n}}{2}\right)\left|\psi_{0}\right\rangle, \quad \tilde{L}(\lambda)\left|\psi_{\mathrm{n}}\right\rangle=\left(\lambda+\frac{i \mathrm{n}}{2}\right)\left|\psi_{\mathrm{n}}\right\rangle
$$

and

$$
\tilde{L}(\lambda)\left|\psi_{k}\right\rangle=\ldots=f_{k} \hat{e}_{1}^{(2)} \otimes \hat{e}_{k+1}^{(\mathrm{n})}\left(\mathcal{A}_{k+1}+\mathcal{B}_{k} y_{k}\right)+f_{k+1} \hat{e}_{2}^{(2)} \otimes \hat{e}_{k}^{(\mathrm{n})}\left(\mathcal{D}_{k}+\mathcal{C}_{k} y_{k}^{-1}\right)=\epsilon_{\mathrm{n}, k}\left|\psi_{k}\right\rangle,
$$

where we have defined $y_{k} \equiv \frac{f_{k+1}}{f_{k}}$. In order for $\left|\psi_{k}\right\rangle$ to be an eigenvector we require the following relation to hold

$$
\mathcal{A}_{k+1}+\mathcal{B}_{k} y_{k}=\mathcal{D}_{k}+\mathcal{C}_{k} y_{k}^{-1}
$$

or equivalently,

$$
\mathcal{B}_{k} y_{k}^{2}+y_{k}\left(\mathcal{A}_{k+1}-\mathcal{D}_{k}\right)-\mathcal{C}_{k}=0 .
$$

Solving this algebraic equation provides us directly with the eigenvalues of the defect operator, which would have the generic expression

$$
\epsilon_{\mathrm{n}, k}^{(1,2)}=\mathcal{A}_{k+1}+\mathcal{B}_{k} y_{k}^{(1,2)} .
$$

Rational case: let us first define the following functions in the rational case

$$
\mathcal{A}_{k}=\lambda+i \alpha_{k}+\frac{i}{2}, \quad \mathcal{B}_{k}=\mathcal{C}_{k}=i C_{k}, \quad \mathcal{D}_{k}=\lambda-i \alpha_{k}+\frac{i}{2} .
$$

Substituting the corresponding functions for the rational case leads to the following values of $y_{k}$

$$
y_{k}^{(1)}=\sqrt{\frac{k}{\mathrm{n}-k}}=-\frac{1}{y_{k}^{(2)}},
$$


implying that the corresponding eigenvalues are

$$
\epsilon_{\mathrm{n}, k}^{(1,2)}=\lambda \pm \frac{i \mathrm{n}}{2}
$$

It is interesting that the eigenvalues are $k$-independent, thus the generic spin- $S$ defect matrix possesses only two distinct eigenvalues, each one being n-fold degenerate.

Trigonometric case: the relevant functions in this case are:

$$
\begin{aligned}
\mathcal{A}_{k} & =\frac{1}{2}\left(e^{\mu\left(\lambda+\frac{i}{2}\right)} q^{\alpha_{k}}-e^{-\mu\left(\lambda+\frac{i}{2}\right)} q^{-\alpha_{k}}\right), \quad \mathcal{B}_{k}=\mathcal{C}_{k}=\frac{1}{2}\left(q-q^{-1}\right) \tilde{C}_{k} \\
\mathcal{D} & =\frac{1}{2}\left(e^{\mu\left(\lambda+\frac{i}{2}\right)} q^{-\alpha_{k}}-e^{-\mu\left(\lambda+\frac{i}{2}\right)} q^{\alpha_{k}}\right) .
\end{aligned}
$$

After some calculations, we find that the eigenvalues in the trigonometric case are much more complicated, and given by the following expression

$$
\begin{aligned}
\epsilon_{\mathrm{n}, k}^{(1,2)}= & \cos \left[\frac{\mu}{2}(\mathrm{n}-2 k)\right] \sinh (\lambda \mu) \\
& \pm \frac{1}{2}[-1-\cos [\mu(2 k-\mathrm{n})]+2 \cos (\mathrm{n} \mu)+\cosh 2 \lambda \mu(-1+\cos [\mu(\mathrm{n}-2 k)])]^{\frac{1}{2}} .
\end{aligned}
$$

Spin eigenvalues: we may also compute the total spin $S_{T}^{z}$ associated to the defect matrix

$$
S_{T}^{z}=\frac{\sigma^{z}}{2} \otimes \mathbb{I}_{\mathrm{n}}+\mathbb{I}_{2} \otimes S^{z},
$$

where $S^{z}$ is given by the $\mathrm{n} \times \mathrm{n}$ matrix (2.4) corresponding to the spin $S$ representation, and $\mathbb{I}_{m}$ are the $m$-dimensional unit matrices. As noted already, the states found above (A.2) are also the $S^{z}$ eigenstates. The spin eigenvalue problem reads then as

$$
\begin{aligned}
S_{T}^{z}\left|\psi_{0}\right\rangle & =\left(S+\frac{1}{2}\right)\left|\psi_{0}\right\rangle, \\
S_{T}^{z}\left|\psi_{k}\right\rangle & =\left(S+\frac{1}{2}-k\right)\left|\psi_{k}\right\rangle, \quad k \in\{1, \ldots, \mathrm{n}-1\} \\
S_{T}^{z}\left|\psi_{\mathrm{n}}\right\rangle & =\left(-S-\frac{1}{2}\right)\left|\psi_{n}\right\rangle .
\end{aligned}
$$

We end up with $2 \mathrm{n}$ eigenstates and eigenvalues. Each eigenvalue $S-k+\frac{1}{2}, k \in\{1, \ldots, \mathrm{n}-1\}$ is 2-fold degenerate, so that in total there are $n+1$ distinct eigenvalues, as expected from the spin summation rules, which take the familiar values: $S_{T}^{z} \in\left\{-S-\frac{1}{2}, \ldots, S+\frac{1}{2}\right\}$.

\section{B Unitarity, crossing symmetry \& Casimir operators}

In this appendix we present the unitarity and crossing symmetry properties of the transmission matrix, and confirm the findings of the present investigation by exploiting these basic requirements together with the fact that the transmission matrix satisfies the quadratic 
algebra (2.33). The unitarity and crossing symmetry are given by the following expressions respectively

$$
\begin{aligned}
\mathbb{T}_{12}(\lambda) \mathbb{T}_{12}(-\lambda) & =\mathbb{I} \\
\mathbb{T}_{12}^{t_{1}}(-\lambda+i) M_{1} \mathbb{T}_{12}^{t_{1}}(\lambda+i) M_{1} & =\mathbb{I},
\end{aligned}
$$

where $t_{1}$ denotes transposition on the first vector space. In both the isotropic and anisotropic case in the principal gradation, which are considered here, $M=\mathbb{I}$.

Rational case: let us first consider the XXX isotropic case. Recall the expression found for the transmission matrix associated to the spin $S$ representation:

$$
\begin{aligned}
\mathbb{T}(\lambda, S) & =\frac{T(\lambda, S)}{i \lambda+S+\frac{1}{2}} \mathbb{M}(\lambda, S) \\
\mathbb{M}(\lambda, S) & =\left(\begin{array}{cc}
i \lambda+S^{z}+\frac{1}{2} & S^{-} \\
S^{+} & i \lambda-S^{z}+\frac{1}{2}
\end{array}\right),
\end{aligned}
$$

where $T(\lambda)$ is defined in $(2.28)$.

The requirement for unitarity leads to:

$$
T(\lambda, S) T(-\lambda, S)=\mathbb{I},
$$

whereas the crossing symmetry condition provides:

$$
T(\lambda+i, S) T(-\lambda+i, S) \frac{\left(i \lambda+S+\frac{1}{2}\right)\left(-i \lambda+S+\frac{1}{2}\right)}{\left(i \lambda+S-\frac{1}{2}\right)\left(-i \lambda+S-\frac{1}{2}\right)}=\mathbb{I} .
$$

To obtain the relations above we have used the following

$$
\begin{aligned}
\mathbb{M}_{12}(\lambda, S) \mathbb{M}_{12}(-\lambda, S) & =\mathbb{M}_{12}^{t_{1}}(\lambda+i, S) \mathbb{M}_{12}^{t_{1}}(-\lambda+i, S) \\
& =\left(\lambda^{2}+C\right) \mathbb{I}=\left(i \lambda+S+\frac{1}{2}\right)\left(-i \lambda+S+\frac{1}{2}\right) \mathbb{I}
\end{aligned}
$$

$C$ is the Casimir operator of $\mathfrak{s l}_{2}$, and one may easily show for the spin $S$ representation that

$$
C=\left(S^{z}\right)^{2}+\frac{1}{2}\left\{S^{-}, S^{+}\right\}+\frac{1}{4}=\frac{(2 S+1)^{2}}{4} .
$$

The latter is valid even for any generic real $S$. A discussion on the spin $S$ (any real) representation of $\mathfrak{s l}_{2}$ expressed in terms of differential operators may be found in e.g. [37] and references therein. It is easily confirmed by inspection that $T$ defined in (2.28) satisfies relations (B.3), (B.4) emanating from the basic properties.

Trigonometric case: similarly for the anisostropic XXZ chain recall the transmission matrix associated to the spin $S$ representation of the deformed algebra

$$
\begin{aligned}
\mathbb{T}(\lambda, \mu, S) & =\frac{T(\lambda, S)}{\sin \left(\mu\left(i \lambda+S+\frac{1}{2}\right)\right)} \tilde{\mathbb{M}}(\lambda, \mu, S) \\
\tilde{\mathbb{M}}_{q}(\lambda, \mu, S) & =\left(\begin{array}{cc}
\sin \left(\mu\left(i \lambda+S^{z}+\frac{1}{2}\right)\right) & \sin (\mu) S^{-} \\
\sin (\mu) S^{+} & \sin \left(\mu\left(i \lambda-S^{z}+\frac{1}{2}\right)\right)
\end{array}\right)
\end{aligned}
$$

$\mu=\pi \gamma, T(\lambda, S)$ defined in (3.17), (3.31). 
As in the isotropic case the unitarity and crossing-unitarity conditions lead to:

$$
\begin{array}{r}
T(\lambda, \mu, S) T(-\lambda, \mu, S)=\mathbb{I}, \\
T(\lambda+i, \mu, S) T(-\lambda+i, \mu, S) \frac{\sin \left(\mu\left(i \lambda+S+\frac{1}{2}\right)\right) \sin \left(\mu\left(-i \lambda+S+\frac{1}{2}\right)\right)}{\sin \left(\mu\left(i \lambda+S-\frac{1}{2}\right)\right) \sin \left(\mu\left(-i \lambda+S-\frac{1}{2}\right)\right)}=\mathbb{I}
\end{array}
$$

Again confirmation of the findings regarding the anisotropic case is easily done by inspection.

The latter relation were obtained using the fact that:

$$
\begin{aligned}
\tilde{\mathbb{M}}_{12}(\lambda, \mu, S) \tilde{\mathbb{M}}_{12}(-\lambda, \mu, S) & =\tilde{\mathbb{M}}_{12}^{t_{1}}(\lambda+i, \mu, S) \tilde{\mathbb{M}}_{12}^{t_{1}}(-\lambda+i, \mu, S) \\
& =\left(-\frac{1}{2} \cos (2 \mu i \lambda)+\frac{1}{4} C_{q}\right) \mathbb{I} \\
& =\sin \left(\mu\left(i \lambda+S+\frac{1}{2}\right)\right) \sin \left(\mu\left(-i \lambda+S+\frac{1}{2}\right)\right) \mathbb{I}
\end{aligned}
$$

$C_{q}$ is the associated $q$-deformed Casimir operator of the $\mathfrak{U}_{q}\left(\mathfrak{s l}_{2}\right)$ algebra $\left(q=e^{i \mu}\right)$, and one may easily show for the spin $S$ representation that

$$
\begin{aligned}
C_{q} & =q q^{2 S^{z}}+q^{-1} q^{-2 S^{z}}+\left(q-q^{-1}\right)^{2} S^{-} S^{+} \\
& =q q^{-2 S^{z}}+q^{-1} q^{2 S^{z}}+\left(q-q^{-1}\right)^{2} S^{+} S^{-}=2 \cos (\mu(2 S+1)) .
\end{aligned}
$$

The results for both rational and trigonometric cases are valid for any real values $S$ of the representation. In this case the representation may be expressed in terms of differential operators (see e.g. [37] and references therein).

\section{The XXZ model: generic values of the spin}

In the main text we have computed the transmission matrices given that the spin parameter takes values in a restricted interval. We generalize here our results for generic spin values. It is clear that these generalizations are due to the periodicity of the involved trigonometric functions.

Repulsive regime: let us introduce the Fourier transform of $a_{y}$ for generic values of $y=2 S$ :

$$
\hat{a}_{y}(\omega)=\frac{\sinh \left(((2 m+1) \nu-y) \frac{\omega}{2}\right)}{\sinh \left(\nu \frac{\omega}{2}\right)}, \quad 2 m \nu<y<2(m+1) \nu .
$$

The effect of the latter generalization has the following effects in our computations in the repulsive regime of the XXZ model: the new "shifted" spin becomes

$$
\tilde{S}=S-m-\frac{1}{2}
$$


whereas the expression for the transmission matrix is again given by (3.19), where

$$
\begin{aligned}
T(\hat{\lambda}, \gamma, \tilde{S})= & \prod_{k=0}^{\infty} \frac{\Gamma\left(\hat{z}+\gamma \tilde{S}-m+\frac{\gamma}{2}+(2 k+1) \gamma\right) \Gamma\left(\hat{z}-\gamma \tilde{S}+m-\frac{\gamma}{2}+(2 k+1) \gamma+1\right)}{\Gamma\left(\hat{z}+\gamma \tilde{S}-m+\frac{\gamma}{2}+2 k \gamma\right) \Gamma\left(\hat{z}-\gamma \tilde{S}+m-\frac{\gamma}{2}+2(k+1) \gamma+1\right)} \\
& \times \frac{\Gamma\left(-\hat{z}+\gamma \tilde{S}-m+\frac{\gamma}{2}+2 k \gamma\right) \Gamma\left(-\hat{z}-\gamma \tilde{S}+m-\frac{\gamma}{2}+2(k+1) \gamma+1\right)}{\Gamma\left(-\hat{z}+\gamma \tilde{S}-m+\frac{\gamma}{2}+(2 k+1) \gamma\right) \Gamma\left(-\hat{z}-\gamma \tilde{S}+m-\frac{\gamma}{2}+(2 k+1) \gamma+1\right)},
\end{aligned}
$$

$\hat{z}=i \hat{\lambda} \gamma$

Attractive regime: first we introduce the generalized Fourier transform for $b_{y}$

$$
\hat{b}_{y}(\omega)=-\frac{\sinh \left((y-2 m \nu) \frac{\omega}{2}\right)}{\sinh \left(\nu \frac{\omega}{2}\right)}, \quad m \nu<y<(m+1) \nu .
$$

Then the transmission matrix in the attractive regime is given by (3.31), where

$$
\begin{aligned}
T(\hat{\lambda}, \gamma, \tilde{S})= & \prod_{k=0}^{\infty} \frac{\Gamma\left(\hat{z}-\xi+m(\gamma+1)+2(k+1) \gamma+\frac{1}{2}\right) \Gamma\left(\hat{z}+\xi-m(\gamma+1)+2 k \gamma+\frac{1}{2}\right)}{\Gamma\left(\hat{z}-\xi+m(\gamma+1)+(2 k+1) \gamma+\frac{1}{2}\right) \Gamma\left(\hat{z}+\xi-m(\gamma+1)+(2 k+1) \gamma+\frac{1}{2}\right)} \\
& \times \frac{\Gamma\left(-\hat{z}-\xi+m(\gamma+1)+(2 k+1) \gamma+\frac{1}{2}\right) \Gamma\left(-\hat{z}+\xi-m(\gamma+1)+(2 k+1) \gamma+\frac{1}{2}\right)}{\Gamma\left(-\hat{z}-\xi+m(\gamma+1)+2(k+1) \gamma+\frac{1}{2}\right) \Gamma\left(-\hat{z}+\xi-m(\gamma+1)+2 k \gamma+\frac{1}{2}\right)}
\end{aligned}
$$

where

$$
\hat{z}=i \hat{\lambda}, \quad \xi=S+\frac{\gamma}{2}, \quad \tilde{S}=m
$$

\section{References}

[1] G. Delfino, G. Mussardo and P. Simonetti, Statistical models with a line of defect, Phys. Lett. B 328 (1994) 123 [hep-th/9403049] [INSPIRE].

[2] G. Delfino, G. Mussardo and P. Simonetti, Scattering theory and correlation functions in statistical models with a line of defect, Nucl. Phys. B 432 (1994) 518 [hep-th/9409076] [INSPIRE].

[3] R. Konik and A. LeClair, Purely transmitting defect field theories, Nucl. Phys. B 538 (1999) 587 [hep-th/9703085] [INSPIRE].

[4] P. Bowcock, E. Corrigan and C. Zambon, Some aspects of jump-defects in the quantum sine-Gordon model, JHEP 08 (2005) 023 [hep-th/0506169] [INSPIRE].

[5] E. Corrigan and C. Zambon, A transmission matrix for a fused pair of integrable defects in the sine-Gordon model, J. Phys. A 43 (2010) 345201 [arXiv:1006.0939] [InSPIRE].

[6] Z. Bajnok and A. George, From defects to boundaries, Int. J. Mod. Phys. A 21 (2006) 1063 [hep-th/0404199] [INSPIRE].

[7] Z. Bajnok, Equivalences between spin models induced by defects,

J. Stat. Mech. 0606 (2006) P06010 [hep-th/0601107] [INSPIRE].

[8] Z. Bajnok and Z. Simon, Solving topological defects via fusion, Nucl. Phys. B 802 (2008) 307 [arXiv:0712.4292] [INSPIRE].

[9] R. Weston, An algebraic setting for defects in the XXZ and sine-Gordon models, arXiv:1006.1555 [INSPIRE]. 
[10] M. Mintchev, É. Ragoucy and P. Sorba, Scattering in the presence of a reflecting and transmitting impurity, Phys. Lett. B 547 (2002) 313 [hep-th/0209052] [INSPIRE].

[11] M. Mintchev, É. Ragoucy and P. Sorba, Reflection transmission algebras, J. Phys. A 36 (2003) 10407 [hep-th/0303187] [InSPIRE].

[12] V. Caudrelier, M. Mintchev and É. Ragoucy, The quantum non-linear Schrödinger model with point-like defect, J. Phys. A 37 (2004) L367 [hep-th/0404144] [INSPIRE].

[13] E. Corrigan and C. Zambon, Jump-defects in the nonlinear Schrödinger model and other non-relativistic field theories, Nonlinearity 19 (2006) 1447 [nlin/0512038].

[14] P. Bowcock, E. Corrigan and C. Zambon, Affine Toda field theories with defects, JHEP 01 (2004) 056 [hep-th/0401020] [INSPIRE].

[15] E. Corrigan and C. Zambon, Comments on defects in the $a_{r}$ Toda field theories, J. Phys. A 42 (2009) 304008 [arXiv:0902.1307] [InSPIRE].

[16] E. Corrigan and C. Zambon, On purely transmitting defects in affine Toda field theory, JHEP 07 (2007) 001 [arXiv:0705.1066] [INSPIRE].

[17] E. Corrigan and C. Zambon, A new class of integrable defects, J. Phys. A 42 (2009) 475203 [arXiv: 0908.3126] [INSPIRE].

[18] V. Caudrelier, On a systematic approach to defects in classical integrable field theories, Int. J. Geom. Meth. Mod. Phys. 5 (2008) 1085 [arXiv:0704.2326].

[19] I. Habibullin and A. Kundu, Quantum and classical integrable sine-Gordon model with defect, Nucl. Phys. B 795 (2008) 549 [arXiv:0709.4611] [INSPIRE].

[20] F. Nemes, Semiclassical analysis of defect sine-Gordon theory, Int. J. Mod. Phys. A 25 (2010) 4493 [arXiv:0909.3268] [inSPIRE].

[21] A. Doikou, Defects in the discrete non-linear Schrödinger model, Nucl. Phys. B 854 (2012) 153 [arXiv:1106.1602] [InSPIRE].

[22] J. Avan and A. Doiku, Liouville integrable defects: the non-linear Schrödinger paradigm, JHEP 01 (2012) 040 [arXiv:1110.4728] [INSPIRE].

[23] J. Avan and A. Doiku, The sine-Gordon model with integrable defects revisited, JHEP 11 (2012) 008 [arXiv:1205.1661] [INSPIRE].

[24] A.R. Aguirre, T.R. Araujo, J.F. Gomes and A.H. Zimerman, Type-II Bäcklund transformations via gauge transformations, JHEP 12 (2011) 056 [arXiv:1110.1589] [INSPIRE].

[25] A.R. Aguirre, Inverse scattering approach for massive Thirring models with integrable type-II defects, J. Phys. A 45 (2012) 205205 [arXiv:1111.5249] [InSPIRE].

[26] A. Doikou and N. Karaiskos, $\sigma$-models in the presence of dynamical point-like defects, Nucl. Phys. B 867 (2013) 872 [arXiv:1207.5503] [INSPIRE].

[27] L.D. Faddeev and L.A. Takhtajan, Spectrum and scattering of excitations in the one-dimensional isotropic Heisenberg model, J. Sov. Math. 24 (1984) 241 [InSPIRE].

[28] L. Faddeev, Algebraic aspects of Bethe ansatz, Int. J. Mod. Phys. A 10 (1995) 1845 [hep-th/9404013] [INSPIRE].

[29] P.P. Kulish and E.K. Sklyanin, Quantum spectral transform method. Recent developments, Lecture Notes in Physics volume 151, Springer, U.S.A. (1982). 
[30] L.A. Takhtajan, Quamtum groups. Introduction to quantum groups and intergable massive models of quantum field theory, in Nankai lectures on mathematical physics, M.-L. Ge and B.-H. Zhao eds., World Scientific, Singapore (1990).

[31] V.E. Korepin, N.M. Bogoliubov, and A.G. Izergin, Quantum inverse scattering method, correlation functions and algebraic Bethe ansatz, Cambridge University Press, Cambridge U.K. (1993).

[32] A. Tsvelik and P.B. Wiegmann, The exact results for magnetic alloys, Adv. Phys. 32 (1983) 331.

[33] N. Andrei and H. Johannesson, Heisenberg chain with impurities (an integrable model), Phys. Lett. A 100 (1984) 108.

[34] H. Frahm and A.A. Zvyagin, The open spin chain with impurity: an exact solution, J. Phys. Cond. Matt. 9 (1997) 9939.

[35] A. Kluemper and A.A. Zvyagin, Disordered magnetic impurities in uniaxial critical quantum spin chains, J. Phys. Cond. Matt. 12 (2000) 8705.

[36] A.A. Zvyagin, Finite size effects in correlated electron models: exact results, Imperial College Press, London U.K. (2005).

[37] A. Doikou, S. Evangelisti, G. Feverati and N. Karaiskos, Introduction to quantum integrability, Int. J. Mod. Phys. A 25 (2010) 3307 [arXiv:0912.3350] [INSPIRE].

[38] Doikou, Bulk and boundary S matrices for the SU(N) chain, Nucl. Phys. B 521 (1998) 547 [hep-th/9803118] [INSPIRE].

[39] N. Andrei and C. Destri, Dynamical symmetry breaking and fractionization in a new integrable model, Nucl. Phys. B 231 (1984) 445 [INSPIRE].

[40] A. Doikou, L. Mezincescu and R. Nepomechie, Factorization of multiparticle scattering in the Heisenberg spin chain, Mod. Phys. Lett. A 12 (1997) 2591 [hep-th/9707155] [INSPIRE].

[41] P.P. Kulish and N. Yu. Reshetikhin, Generalized Heisenberg ferromagnet and Gross-Neveu model, Zh. Eksp. Teor. Fiz. 80 (1981) 214.

[42] A. Doikou, L. Mezincescu and R. Nepomechie, Simplified calculation of boundary S matrices, J. Phys. A 30 (1997) L507 [hep-th/9705187] [InSPIRE].

[43] P. Kulish and N.Y. Reshetikhin, Quantum linear problem for the sine-Gordon equation and higher representation, J. Sov. Math. 23 (1983) 2435 [INSPIRE].

[44] A. Doikou and R. Nepomechie, Direct calculation of breather $S$ matrices, J. Phys. A 32 (1999) 3663 [hep-th/9903066] [InSPIRE].

[45] A.B. Zamolodchikov and A.B. Zamolodchikov, Factorized s Matrices in Two-Dimensions as the Exact Solutions of Certain Relativistic Quantum Field Models, Annals Phys. 120 (1979) 253 [INSPIRE].

[46] A. Doikou and R. Nepomenchie, Soliton $S$ matrices for the critical $A(1)(N-1)$ chain, Phys. Lett. B 462 (1999) 121 [hep-th/9906069] [INSPIRE].

[47] L.A. Takhtajan and L.D. Faddeev, The quantum inverse problem method and the XYZ Heisenberg model, Russian Math. Surv. 34 (1979) 13.

[48] L.A. Takhtajan, The quantum inverse problem method and the XYZ Heisenberg model, Physica D 3 (1981) 231. 